\title{
ATENCIÓN QUIRÚRGICA EN HOMBRES Y MUJERES ¿DIFERENTE O DESIGUAL?
}

\author{
Charlene Beth Arnold Bichler ${ }^{1}$, María Teresa Ruiz Cantero ${ }^{2}$, Jordi Torrubiano \\ Domínguez ${ }^{3}$, Vicente Clemente Gómez ${ }^{4}$ y Teresa Blasco Segura ${ }^{5}$ \\ carlabel@telefonica.net
}

Recibido: 29-01-2010

Aceptado: 22-04-2010

\section{Resumen}

Explorar la posible existencia de sesgos de género en procedimientos quirúrgicos (PQ) frecuentes $\mathrm{y}$ en sus estancias medias (EM) postquirúrgicas es objetivo del presente trabajo, mediante su comparación entre sexos en el Servicio de Cirugía General y Digestiva del Hospital General de Alicante (20002004). Se intervienen más hombres que mujeres en 4 de los 7 PQ estudiados, apuntando posibles sesgos de género en el patrón quirúrgico, como por apendicectomías agudas (1,36 Hombres/1 Mujer) y complicadas (1,79/1). La estancia postapendicectomías, a igual edad y comorbilidad, es de 2 días más en hombres $(9,49)$ que en mujeres $(7,5)$. No detectar diferencias por sexo en colecistectomías por colecistitis (más frecuentes en mujeres), puede indicar colelitiasis evolucionadas por no sospecha diagnóstica en hombres.

Palabras clave: Sesgo de género, cirugía, desigualdades en salud, procedimientos quirúrgicos del sistema digestivo, estancia media tras cirugía.

\footnotetext{
${ }^{1}$ Hospital General Universitario de Alicante y Universidad de Alicante.

${ }^{2}$ Universidad de Alicante y CIBER Epidemiología y Salud Pública.

${ }^{3}$ Universidad de Alicante y Salud Pública.

${ }^{4}$ Universidad de Alicante y CIBER Epidemiología y Salud Pública.

${ }^{5}$ Hospital General Universitario de Alicante.
} 


\begin{abstract}
The purpose of this work is to explore the possible existence of gender bias in frequent surgical procedures (SP) and its postoperative median length of stay (LOS), through comparison between both sexes in the Gastrointestinal and General Surgery Unit of the Alicante General Hospital (2000-2004). More men than women are operated in 4 of the 7 SP studied, aiming at possible gender bias in surgical patterns, as about of acute (1.36 Men/1 Woman) and complicated (1.79/1) appendectomies. With the same age and morbidity, post-appendectomy LOS is 2 days higher in men (9.49) than in women (7.5). Not detecting sex differences in cholecystectomies due to cholecystitis (more frequent in women), may suggest well-established cholelithiasis because of unsuspected diagnosis in men.
\end{abstract}

Key words: Gender bias, surgery, health inequalities, digestive system surgical procedures, length of stay (LOS).

\title{
1. Introducción
}

Desde comienzos de los años 90 los estudios sobre sesgos de género en el sector sanitario han trabajado sobre problemas de salud concretos (Ruiz Cantero y Verbrugge, 1997; Ruiz Cantero et al., 2007), mostrando su existencia en el manejo de la hipertensión (Garavaglia et al., 1989; Schwartzbauer y Robbins, 2001), infartos de miocardio y aneurismas aorticos (Aguilar, Lázaro, Fitch y Luengo, 2002; Hollenbeak, Weisman, Rossi y Ettinger, 2006), EPOC (Baibergenova et al., 2006), obesidad (Zizza, Herring, Stevens y Carey, 2003), dislipemias (Gervás y Pérez Fernández, 1997), diabetes (Baker, Eriksson, Forsen y Osmond, 2005), infecciones (Ruiz Cantero y Verdú-Delgado, 2004) y cáncer (González et al., 2005; Beresfold, Weiss, Voigt y McKnight, 1997). 
Uno de los determinantes de las desigualdades (variaciones injustas y evitables) en salud planteado por la OMS es el propio sector sanitario, pues puede originar diferencias evitables en el acceso, uso y calidad asistencial según la clase social, el sexo y la etnia de quienes padecen (ONU, 2001). La cobertura sanitaria es universal en España, por tanto, dentro del sector sanitario no deberían existir barreras de acceso. No obstante, se ha evidenciado una mayor demora y espera en la atención sanitaria de las mujeres respecto a los hombres (Ruiz Cantero y Verdú-Delgado, 2004). Esto sucede en los servicios de Urgencias, donde a igual problema de salud las mujeres esperan más tiempo para ser atendidas (Ruiz Cantero et al., 1995). Al contrario, en las Urgencias pediátricas ingresan más niños que niñas (Rajmil et al., 1999).

En cirugía, se ha evidenciado también la existencia de sesgos de género, al observarse menos intervenciones en mujeres que en hombres a igual necesidad (Johnston, 1994; Javitt et al., 1995). El Institut Català d' Oncologia mostró que las mujeres son menos readmitidas que los hombres por cáncer colorrectal avanzado, una vez controlado por características del tumor, mortalidad y comorbilidad (González et al., 2005). Estos resultados son relevantes, pues si las diferencias en el tratamiento quirúrgico según el sexo no se fundamentan en razones clínicas, las variaciones en los patrones de consumo de recursos hospitalarios podrían ser injustas (Bernard, Hayward, Rosevear y McMahon, 1993; Austin, Rothwell y Tu, 2002).

Las diferencias en la prevalencia según sexo y la gravedad clínica influyen en los esfuerzos diagnósticos y terapéuticos. Por ello, para afirmar que existen sesgos de género es necesario recoger siempre información sobre ambas características, de tal forma que a igual prevalencia y gravedad no debieran desarrollarse diferentes estrategias diagnósticas y terapéuticas (Ruiz Cantero y Verdú-Delgado, 2004). Probablemente debido a que los análisis de género han de incorporar desde sus diseños las diferencias en las prevalencias entre sexos no siempre conocidas, y la gravedad, se ha producido escaso conocimiento sobre la posible existencia de estos sesgos en las unidades quirúrgicas (Austin, Rothwell y Tu, 2002). 
Fuera de España la literatura científica que muestra la identificación de sesgos de género en el esfuerzo terapéutico quirúrgico ha analizado comparativamente la frecuencia de intervenciones en ambos sexos, según edad y gravedad (Javitt et al., 1995; Austin, Rothwell y Tu, 2002; Carbonell et al., 2005). También, se han estudiado los ingresos hospitalarios y la estancia no justificados, según edad y comorbilidad (Sangha et al., 2002). No obstante, se precisa información sobre la eventual existencia de desigualdades de género en las estancias post-quirúrgicas que son en definitiva las de responsabilidad de una unidad de cirugía (Austin, Rothwell y Tu, 2002; Carbonell et al., 2005; Ruiz López et al., 2005).

El objetivo, de este trabajo es explorar la posible existencia de sesgos de género en procedimientos quirúrgicos de Cirugía General y Digestivo frecuentes, como las apendicectomías, colecistectomías, intervenciones por hernias y resecciones por neoplasias benignas y malignas de intestino y recto, mediante la comparación según sexo de las frecuencias de estos procedimientos y de sus estancias medias postquirúrgicas, en un hospital público de nivel terciario en España, como es el Hospital General Universitario de Alicante, durante el período 2000-2004.

\section{Material y Métodos}

Se realizó un estudio descriptivo de carácter transversal de las intervenciones quirúrgicas programadas y urgentes realizadas durante el quinquenio 2000-2004 en el Servicio de Cirugía General y Digestiva del Hospital General Universitario de Alicante (HGUA), que atiende a la población del Departamento 19 de Salud de la Comunidad Valenciana (Alicante) y de referencia de la provincia. En concreto, la población estudiada son 4.836 (2.284 hombres y 1.552 mujeres) personas mayores de 14 años a quiénes se les practicaron apendicectomías (1. apendicitis aguda, o 2. apendicitis con absceso intrabdominal y/o peritonitis), intervenciones de hernias (1. inguinocrurales, o 2. ventrales, incisionales y de pared abdominal), colecistectomías (1. litiasis de vesícula 
y vías biliares sin colecistitis, o 2. con colecistitis), y resecciones por neoplasias benignas y malignas de intestino y recto. Se han seleccionado estos procedimientos quirúrgicos por atender a procesos de elevada frecuencia poblacional y de atención en la especialidad estudiada. Esta población (Anexo I) se obtuvo después de excluir los procedimientos quirúrgicos que no se justificaban en función de los procesos diagnósticos adjudicados en el CMBD ( $n=576 ; 306$ hombres y 270 mujeres).

La fuente de información corresponde a los datos informatizados del CMBD (CIE-9-MC) facilitados por la Unidad de Documentación Clínica y Admisión del HGUA. Las variables recogidas son: edad, sexo, diagnóstico principal, 4 diagnósticos secundarios, procedimiento quirúrgico, y fechas de intervención y de alta. Ambas fechas sirvieron para crear la variable denominada estancia media post-quirúrgica. Por otra parte, se utilizó el Registro General del Sistema de Información Poblacional (Tarjetas SIP) del Departamento 19 de Salud de la Comunidad Valenciana para calcular las tasas poblacionales de procedimientos quirúrgicos por sexo. Debido a que este Registro tiene datos desde 2000 en esta área, el período de estudio se acotó a los casos intervenidos en el quinquenio 2000 a 2004.

Para las intervenciones de hernias y colecistectomías la edad se reagrupó entre pacientes de 15 a 54 años y de 55 en adelante. Los pacientes de apendicectomías se estratificaron de 15 a 34 años y de 35 años en adelante por la mayor frecuencia de apendicitis en adolescentes y adulto joven. Y los pacientes con resecciones por neoplasias de intestino y recto se estratificaron en 3 categorías: 15-64 años, 65 a 75 años y mayores de 75 años por ser más prevalentes a mayor edad.

Los diagnósticos secundarios se clasificaron en 3 grupos según el CIE-9-MD ( $\mathrm{M}^{\mathrm{o}}$ de Sanidad y Consumo, 2008): 1. Comorbilidad por diagnósticos que se desarrollan a lo largo de la estancia hospitalaria e influyen en la duración de la misma o en el tratamiento administrado: 1.a. Complicaciones quirúrgicas (CQ) y 1.b. Complicaciones diagnósticas derivadas de afecciones independientes ocurridas durante el ingreso hospitalario (CD). Y 2. Comorbilidad general (CG) debida a diagnósticos que no siendo el principal coexisten con él en el momento del ingreso (Anexo II). La variable sexo de 
quien padece fue considerada como la principal variable explicativa, y los procedimientos quirúrgicos y las estancias medias post-quirúrgicas hospitalarias las variables resultado.

Se realizó un estudio sobre las frecuencias de los procedimientos quirúrgicos seleccionados en función del sexo y la edad. En concreto, se calculó la razón entre sexos de los mismos, y las Tasas por sexo de cada procedimiento quirúrgico; así como las Tasas por sexo y edad, y sus correspondientes IC (95\%). Se calcularon las probabilidades (Odds ratio cruda con IC95\%) de intervención quirúrgica en función del sexo tomando como grupo de referencia a la población-tiempo ( 5 años) susceptible de padecer las patologías cuyos tratamientos son los procedimientos quirúrgicos seleccionados. También se calcularon las OR ajustadas por edad. Al no poder asumir normalidad en todos los casos, se utilizó el contraste de Mann-Whitney para identificar la posible existencia de diferencias de los procedimientos quirúrgicos según sexo.

Para analizar las diferencias en la frecuencia de la comorbilidad por sexo, se compararon los porcentajes en hombres y mujeres de cada uno de los 4 diagnósticos secundarios agregados al diagnóstico principal [Chi-cuadrado $\left(\mathrm{X}^{2}\right)$ ] en cada uno de los procedimientos quirúrgicos estudiados. Esta información fue utilizada para identificar si la comorbilidad influye en la estancia media post-quirúrgica. Para lo cual además, se compararon los porcentajes en hombres y mujeres $\left(\mathrm{X}^{2}\right)$ de padecimientos de comorbilidad general, quirúrgica y/o por otros diagnósticos tras ingreso en cada uno de los procedimientos quirúrgicos estudiados.

Se calcularon las medias, medianas y desviación de los días de estancia postquirúrgica según el sexo y edad. Se utilizó el Contraste de Mann-Whitney para comparar los días de estancia media post-quirúrgicos en ambos sexos.

Para el procesamiento y análisis de la información se utiliza el programa SPSS 14.0 . 


\section{Resultados}

\subsection{Frecuencia de los procedimientos quirúrgicos de cirugía general y digestiva según el sexo de las personas intervenidas}

En la Tabla 1 (anexa) se pueden observar la distribución de las frecuencias de los procedimientos quirúrgicos estudiados en función de las patologías; así como la ratio por sexo de los mismos. También se pueden observar las Tasas de procedimientos quirúrgicos por sexo y edad x 10.000 habitantes. Se intervienen más hombres que mujeres en 4 de las 7 patologías estudiadas. Destacan las tasas de intervenciones por hernias inguinocrurales más frecuentes en los hombres que en mujeres $\left(19,56 \times 10^{-4}\right.$ vs. $\left.3,49 \times 10^{-4}\right)$ de todas las edades $(\mathrm{p}<0,001)$. Al eliminar el efecto de la edad (Tabla 2 anexa), la probabilidad de que los hombres sean intervenidos es 4,6 veces superior a que sean intervenidas las mujeres $[\mathrm{OR}=0,16(0,14-0,19)]$.

Las tasas de apendicectomías por apendicitis aguda son significativamente más frecuentes en hombres jóvenes $\left[16,57 \times 10^{-4}\left(14,7 \times 10^{-4}-18,44 \times 10^{-4}\right)\right]$ respecto a mujeres jóvenes $\left[11,84 \times 10^{-4}\left(10,24 \times 10^{-4}-13,44 \times 10^{-4}(\mathrm{p}<0,001)\right.\right.$, aunque también se detectan diferencias significativas por sexo en adultos $(\mathrm{p}=0,01)$. Lo mismo sucede para las apendicectomías por abscesos intrabdominales y/o peritonitis, más frecuentes en hombres adultos $\left[2,87 \times 10^{-4}\left(2,24 \times 10^{-4}-3,49 \times 10^{-4}\right)\right]$ respecto a mujeres del mismo grupo de edad $\left[1,22 \times 10^{-4}\left(0,84 \times 10^{-4}-1,6 \times 10^{-4}\right)\right](\mathrm{p}<0,001)$, y con menor fuerza de la asociación en jóvenes ( $\mathrm{p}=0,04)$ (Tabla 1). Al eliminar el efecto de la edad (Tabla 2) la probabilidad de que los hombres sean intervenidos de apendicitis es el 54\% veces superior a que sean apendicectomizadas las mujeres [OR=0,67 $(0,59-0,76)]$.

También son los hombres a los que mas resecciones por neoplasias benignas y malignas de intestino y recto se les realiza, pero en concreto se detectan diferencias significativas por sexo en los pacientes mayores de 75 años: hombres $\left[25,81 \times 10^{-4}\right.$ $\left.\left(20,02 \times 10^{-4}-31,6 \times 10^{-4}\right)\right]$ y mujeres $\left[13,75 \times 10^{-4}\left(10,46 \times 10^{-4}-17,04 \times 10^{-4}\right)\right](\mathrm{p}<0,001)$ (Tabla 1). Al eliminar el efecto de la edad, la probabilidad de que los hombres sean 
intervenidos es el $21 \%$ superior que de ser intervenidas las mujeres [OR=0,69 $(0,56-$ 0,84)] (Tabla 2) (anexa).

Por el contrario, las tasas de colecistectomías por litiasis de vesícula y vías biliares sin colecistitis son más frecuentes en mujeres $\left[8,31 \times 10^{-4}\left(7,50 \times 10^{-4}-9,12^{-4}\right)\right]$

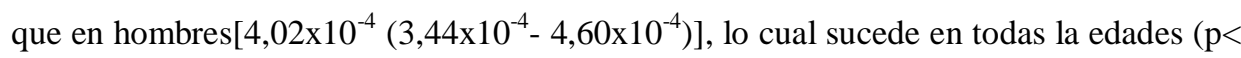
0,001); así como las tasas de intervenciones por hernias ventrales, incisionales y de pared, que son más frecuentes en mujeres especialmente a partir de los 55 años $\left[10,04 \times 10^{-4}\left(8,47 \times 10^{-4}-11,61 \times 10^{-4}\right)\right]$ que en hombres $\left[6,77 \times 10^{-4}\left(5,33 \times 10^{-4}-8,21 \times 10^{-}\right.\right.$

$\left.\left.{ }^{4}\right)\right](\mathrm{p}=0,004)($ Tabla 1). Al eliminar el efecto de la edad, las mujeres tienen una probabilidad mayor (37\%) que los hombres de ser intervenidas $(\mathrm{p}=0,01)$ (Tabla 2).

\subsection{Comorbilidad quirúrgica en hombres y mujeres}

La frecuencia de la comorbilidad por sexo (Diagnóstico principal y 4 secundarios) por procedimiento quirúrgico se reflejan en la Tabla 3 (anexa), y el tipo de comorbilidad de los pacientes por sexo según el procedimiento quirúrgico en la Tabla 4 (anexa). Esto es importante para determinar si la mayor estancia media en un sexo respecto a otro se relaciona con la comorbilidad. Con solo el diagnóstico principal hay significativamente más mujeres que hombres en las colecistectomías por litiasis biliar sin colecistitis $(46,8 \%$ de hombres y $56,7 \%$ de mujeres $)(p=0,02)$. En la Tabla 4 se puede observar que los hombres presentan con mayor frecuencia otros diagnósticos tras ingresar por esta causa $(16,3 \%)$ que las mujeres $(9,4 \%)(p=0,01)$.

Con un diagnóstico secundario más el principal (Tabla 3), hay más mujeres que hombres ingresados para colecistectomías por litiasis biliar con colecistitis $(22,3 \%$ hombres vs. $30,3 \%$ mujeres $)(\mathrm{p}=0,02)$. Aunque por el contrario, hay más hombres $(16,42 \%)$ que mujeres $(7,6 \%)$ con 4 diagnósticos secundarios más el principal $(\mathrm{p}=0,0008)$. En concreto, en la Tabla 4 se observa que las mujeres presentan con mayor frecuencia otros de diagnósticos tras el ingreso $(18,9 \%)$ respecto a los hombres $(12,04 \%) \quad(p=0,02)$. Mientras que los hombres presentan mayor complicación 
quirúrgica $(15,7 \%)$ que las mujeres $(8,2 \%)(\mathrm{p}=0,004)$ y más comorbilidad general $(83,9 \%)$ que estas $(70,03 \%)(\mathrm{p}<0,001)$. Esto último sucede en los mayores de 55 años: $17,3 \%$ de los hombres presentan complicaciones quirúrgicas frente al 7,6\% de las mujeres $(\mathrm{p}=0,02)$ y $92,8 \%$ de los hombres presentan comorbilidad general frente al $78,8 \%$ de las mujeres $(\mathrm{p}<0,001)$.

Con un diagnóstico secundario más el principal (Tabla 3), hay más mujeres que hombres en las intervenciones de hernias inguinocrurales (13,06\% de hombres vs. $20 \%$ de mujeres) $(\mathrm{p}=0,01)$. Son las mujeres mayores de 55 años (Tabla 4) (anexa), las que presentan con mayor frecuencia $(2,9 \%)$ que los hombres $(0,5 \%)$ otros diagnósticos tras el ingreso por estas intervenciones $(\mathrm{p}=0,02)$. No obstante, es un pequeño número de pacientes quienes padecen estos otros diagnósticos. Lo mismo sucede en el caso de las apendicectomías por apendicitis agudas $(\mathrm{p}=0,001)$, pues se observa que un pequeño número de mujeres presentan otros diagnósticos con mayor frecuencia tras el ingreso $(5,4 \%)$ que de hombres $(1,4 \%)$.

Con un diagnóstico secundario más el principal (Tabla 3), también hay más mujeres que hombres en las resecciones por neoplasias de intestino y recto $(17 \%$ hombres vs. $26,9 \%$ mujeres) $(\mathrm{p}=0,01)$.

\subsection{Diferencias en las estancias medias hospitalarias según el sexo en los diferentes procedimientos quirúrgicos}

Respecto a las estancias medias (Tabla 5) (anexa), los hombres mayores de 35 años permanecen más tiempo ingresados que las mujeres por apendicectomías por apendicitis con absceso y/o peritonitis $\left[X_{H}=9,5\right.$ días vs. $X_{M}=7,5$ días $](p<0,006)$, y por colecistectomías por litiasis de vesícula y vías biliares con colecistitis en todas las edades $\left[\mathrm{X}_{\mathrm{H} 15-54 \text { años }}=5,7\right.$ días vs. $\mathrm{X}_{\mathrm{M} 15-54 \text { años }}=4,9$ días $](\mathrm{p}=0,02), \mathrm{y}\left[\mathrm{X}_{\mathrm{H}>55 \text { años }}=11,7\right.$ días vs. $\mathrm{X}_{\mathrm{M}>55 \text { años }}=9,35$ días] $(\mathrm{p}=0,01)$.

Por el contrario, las mujeres mayores de 55 años intervenidas de hernia inguinocrural tienen una media de estancia mayor que los hombres de $\left[\mathrm{X}_{\mathrm{M}}=3,8\right.$ días vs. 
$\mathrm{X}_{\mathrm{H}}=3,4$ días] $(\mathrm{p}=0,007)$. Así mismo, en las intervenciones de hernias ventrales, incisionales y de pared abdominal permanecen mas días de estancia las mujeres que los hombres entre 15 a 54 años $\left[\mathrm{X}_{\mathrm{M}}=5,9\right.$ días vs. $\mathrm{X}_{\mathrm{H}}=4,6$ días $](\mathrm{p}=0,001)$.

\section{Discusión}

\subsection{Las intervenciones más frecuentes tienen perspectiva de género}

Las colecistectomías, la cirugía por hernias y las apendicectomías son las intervenciones más frecuentes en el servicio de cirugía general del Hospital General de Alicante, datos consistentes con el volumen de procedimientos hospitalarios del sistema sanitario español (Librero et al., 2005).

La frecuencia de apendicectomías es mayor en hombres y esto se puede relacionar con la prevalencia poblacional de las apendicitis. No obstante, aunque la prevalencia de apendicitis explique la prevalencia de apendicectomías, lo contrario también es cierto, pues solo post-apendicectomía se confirma el diagnóstico de apendicitis. Esto significa que se desconoce la verdadera prevalencia de las apendicitis según sexo, pues aunque algunas apendicectomías son blancas con mayor frecuencia en mujeres que en hombres (Librero et al., 2005; Flum, Morris, Koepsell y Dellinger, 2001), ciertas apendicitis no son intervenidas.

En este estudio se ha observado que las apendicectomías por apendicitis complicadas (con absceso intraabdominal y/o peritonitis) son el doble en hombres que en mujeres, mientras que por apendicitis agudas son tres hombres por cada dos mujeres. Para que las apendicitis agudas no deriven en complicadas, se acepta el criterio de que alrededor del $25 \%$ de las apendicectomías en mujeres y del $10 \%$ en hombres sean blancas (Librero et al., 2005; Flum, Morris, Koepsell y Dellinger, 2001), porcentajes que disminuyen en las mujeres con la utilización de tomografía axial computarizada, aunque no en hombres (Antevil, Rivera, Langenberg y Brown, 2004). En este sentido, es posible que la reducción de las diferencias en las frecuencias por sexo en las 
apendicectomías complicadas observada en este estudio se deba a que, influidos por la mayor frecuencia de apendicectomías blancas en las mujeres, los profesionales sospechen con menor frecuencia el diagnóstico de apendicitis agudas en ciertas quejas de las mujeres, que derive a su infra-diagnóstico, y con ello a un posterior incremento de las apendicitis complicadas en las mismas.

\subsection{Sesgos de género en los tiempos de permanencia post-operatoria. La comorbilidad como factor explicativo de los esfuerzos terapéuticos quirúrgicos diferenciales}

Las estancias medias por sexo en las apendicectomías coinciden con los resultados de ámbito nacional (Ruiz López et al., 2005; Mº Sanidad y Consumo, 2006). Los hombres adultos (mayores de 35 años) permanecen ingresados 2 días más que las mujeres en las apendicectomías complicadas; lo que podría entenderse como un sesgo de género de la atención post-quirúrgica, salvo que en futuros estudios se constate que puede ser debido a mayor número de complicaciones quirúrgicas en los hombres. En el presente estudio no se han detectado diferencias significativas probablemente por el escaso número de casos complicados. De hecho, son las mujeres quienes presentan significativamente más que los hombres otros diagnósticos en el ingreso para apendicectomías por apendicitis aguda y están menos tiempo ingresadas.

Está descrito que las intervenciones quirúrgicas por hernias presentan una variabilidad geográfica entre moderada y alta (Librero et al., 2005). Las intervenciones por hernias inguinocrurales hospitalizadas son mas frecuentes en hombres, coincidiendo con los hallazgos para el resto de España ( $\mathbf{M}^{\mathrm{o}}$ de Sanidad y Consumo, 2006; Gobierno Vasco, 2000), lo cual se relaciona con su mayor prevalencia (Primatesta y Goldacre, 1996).

Las hernias ventrales dependen del número de intervenciones previas realizadas en la cavidad abdominal (aneurismas de aorta, cirugía cardiaca, transplantes renales, histerectomías o cirugía sucia por graves peritonitis), del tipo de incisión y la 
técnica realizada, y de las infecciones o dehiscencias que ocasionan estas heridas quirúrgicas. Las Tasas estandarizadas por sexo de estas intervenciones oscilan entre áreas de salud (Librero et al., 2005), siendo su variabilidad alta en España y en el ámbito internacional ( $\mathrm{M}^{\mathrm{o}}$ de Sanidad y Consumo, 2006; Gobierno Vasco, 2000; Bucknall, Cox y Ellis, 1982; Harding, 1983). En contra de lo esperado, en este estudio se intervienen a más mujeres que hombres por hernias ventrales. Es en contra de lo esperado porque son los hombres los que con mayor frecuencia son intervenidos de los procesos de cavidad abdominal antes mencionados, exceptuando las histerectomías, y por lo tanto los que más herniaciones deben sufrir (Harding, Mudge, Leinster y Hughes, 1983). La relevancia de la discrecionalidad médica y quirúrgica se apunta como factor explicativo de la variabilidad en estos casos (Aguiló, 2005).

Coincidiendo con las estadísticas nacionales, las mujeres permanecen más días de estancia por cirugía de hernias ( $\mathbf{M}^{\mathrm{o}}$ de Sanidad y Consumo, 2006; Gobierno Vasco, 2000); siendo llamativo el caso de las hernias ventrales. Este hallazgo podría explicarse porque en las mujeres suele producirse una mayor desestructuración anatómica que precisa mayor reparación quirúrgica, cuidados y tiempo (Primatesta y Goldacre, 1996). La mayor estancia post-operatoria en las mujeres mayores de 55 años intervenidas por hernias inguino-crurales puede relacionarse con un incremento de la morbilidad por una menor sospecha y atención diagnóstica en ellas, que tiene como consecuencia una mayor demora en su derivación sanitaria y atención quirúrgica.

La colecistectomía es la intervención más frecuente en mujeres en cirugía general y digestiva coincidiendo con su mayor prevalencia de litiasis biliar (Aguiló, 2005; Schirmer, Winters y Edlich, 2005). No obstante, la prevalencia puede ser aún mayor pues el $40 \%$ de las litiasis biliares son asintomáticas (Schirmer, Winters y Edlich, 2005; Attili et al., 1995). Además, tanto la prevalencia como las intervenciones quirúrgicas dependen de la variabilidad diagnóstica (ecografías) (Martínez de Pancorbo et al., 1997; Attili et al., 1995). Esta se ha mostrado influida por la sospecha diagnóstica -mas frecuente en mujeres- y las decisiones clínicas en situaciones de incertidumbre, por los sistemas de financiación de los servicios sanitarios ( $\mathrm{M}^{\mathrm{o}}$ Sanidad y Consumo, 
2008), y por la introducción de nuevas tecnologías (laparoscopia, cirugía sin ingreso) (Ortún, Puig-Junoy y Callejón, 2005).

Las colecistitis son una complicación de la evolución de la litiasis biliar, siendo su incidencia de alrededor del 3,5\% de las mismas (Tellado et al., 2005). En este sentido, llama la atención que la frecuencia de colecistectomías por litiasis con colecistitis, que es una cirugía urgente en la mayoría de los casos, sea tan similar en ambos sexos, cuando la frecuencia de colecistectomías por litiasis sin colecistitis es tan significativamente mayor en mujeres que hombres. Dado que se observa que la edad no influye, bien podría deberse a la dificultad para diagnosticar las litiasis sin colecistitis en los hombres debido a su mayor comorbilidad general, o a que no se sospecha este problema en ellos por su menor frecuencia, lo que sería un sesgo de género en su perjuicio y que derivará en un incremento de la litiasis con colecistitis. O bien podría sospecharse la existencia de un sesgo de género en la selección previa a la cirugía de esta patología, en el sentido de que las mujeres o sus médicos optan por un tratamiento clínico (Badia y Williamson, 2004).

Se ha observado una mayor estancia media por colecistectomía en hombres que en mujeres en España ( $\mathrm{M}^{\mathrm{o}}$ de Sanidad y Consumo, 2006; Gobierno Vasco, 2000), lo cual aumenta con la edad, pero es ésta y no el sexo la que predice un incremento de la morbi-mortalidad por esta causa (Carbonell et al., 2005), que justificaría la mayor estancia media de los hombres. De acuerdo con ello, este estudio evidencia una significativa mayor estancia media en hombres que en mujeres por colecistectomías por litiasis biliares con colecistitis. Las diferencias por sexo en la comorbilidad pueden explicar este hallazgo, en la medida que aunque las mujeres presentan mayor frecuencia de otros diagnósticos tras el ingreso, los hombres presentan una mayor comorbilidad general y complicaciones quirúrgicas. Esto indicaría un sesgo de género en perjuicio de los hombres por su retraso diagnóstico que complica su postoperatorio (Ruiz Cantero y Verdú-Delgado, 2004; Carbonell et al., 2005).

Las diferencias detectadas por sexo en las resecciones por neoplasias benignas y malignas de intestino y recto aumentan con la edad, puesto que se duplica en número 
de intervenciones en hombres que en mujeres a partir de los 75 años, lo cual coincide con los estándares nacionales publicados (López-Abente et al., 2004). El origen es múltiple, pero principalmente el cáncer colorrectal es la patología que predomina, la cual es más prevalente en hombres (Viñes, Ardanaz, Aráosla y Gaminde, 2003).

\subsection{Limitaciones y retos de los estudios de sesgos de género en Cirugía General y Digestiva}

Los resultados deben ser interpretados considerando las características específicas de los hospitales públicos en España, como las modificaciones en la oferta y gestión de la actividad asistencial: redistribución de procesos a otras especialidades quirúrgicas que durante el tiempo de estudio se han ampliado (cirugía pediátrica), incorporación de técnicas quirúrgicas de hospital terciario (laparoscopia, unidades de cirugía colorrectal, bariátrica, endocrina, hepática, esofágica y pancreática), y contratación de prestación de servicios de cirugía menor y media, remitiendo pacientes a centros privados (plan de choque lista de espera quirúrgica de la comunidad valenciana) (Marqués, Peiró y Medrano Heredia, 2003).

El CMBD no tiene incorporada ninguna clasificación de gravedad que pudiera justificar la mayor estancia media post-quirúrgica en un sexo respecto a otro. Una posibilidad es la utilización del nivel de gravedad asociado a la comorbilidad (índice de Charlson, Elixhauser), sin embargo, siendo ambos buenos predictores de morbimortalidad a un año o más tras ingreso hospitalario por causa médica fundamentalmente, no lo son por causa quirúrgica (Quan et al., 2005; Elixhauser, Steiner, Harris y Coffey, 1998). Para este estudio, se ha preferido cuantificar el número de diagnósticos secundarios añadidos al diagnóstico principal y el tipo de comorbilidad según sexo. 


\section{Conclusiones}

El patrón asistencial en la unidad de Cirugía General y Digestiva del Hospital de Alicante presenta variaciones por sexo, algunas de las cuales apuntan a la existencia de sesgos de género en la atención quirúrgica, una vez ajustados los hallazgos por edad. La colecistitis es una complicación de la litiasis biliar, la cual es más prevalente en mujeres. Por tanto, la no detección de diferencias por sexo en la frecuencia de colecistectomías por litiasis biliar con colecistitis después de ajustar por edad, va en contra de lo esperado. Las estancias medias post-quirúrgicas por esta causa son mayores en hombres que en mujeres, lo que podría deberse a que presentan el doble de complicaciones quirúrgicas y una ligera mayor comorbilidad general, aunque las mujeres presentan significativamente más otros tipos de diagnósticos durante el ingreso. La mayor prevalencia de apendicectomías blancas en mujeres puede inducir a una menor sospecha diagnóstica de apendicitis agudas en éstas que derivará posteriormente en un incremento de apendicitis complicadas. Además, los hombres permanecen más días de estancia en el hospital que las mujeres por esta causa. La no detección de razones clínicas, como una mayor comorbilidad en hombres que en mujeres, hacen pensar en la posible existencia de un sesgo de género. En contra de lo esperado, son las mujeres las más frecuentemente intervenidas de hernias ventrales. Sorprende, pues los hombres son quienes más herniaciones deberían sufrir producidas por el mayor número de intervenciones previas realizadas en la cavidad abdominal.

El todavía escaso conocimiento existente sobre las razones de las variaciones por sexo en las frecuencias y estancias medias de los procedimientos quirúrgicos, aumenta la probabilidad de juicios clínicos basados en sospechas diagnósticas más que en evidencias, especialmente en los pródromos de las enfermedades. Desde los análisis de la semiótica médica se apunta que los profesionales atribuyen diferentes significados a los mismos síntomas y signos según los manifiesten hombres o mujeres (Malterud, 2000). La misma autora enfatiza el rol del intérprete en la interpretación de los síntomas y signos en los procesos diagnósticos; pues así como una misma partitura puede ser mal 
interpretada o con virtuosismo, la interpretación de los síntomas y signos clínicos, y su posterior esfuerzo terapéutico clínico o quirúrgico, depende del conocimiento existente y la habilidad de los profesionales. Estos hechos no pueden suponer la aparición de mecanismos defensivos sobre el quehacer cotidiano de los especialistas que en definitiva pretenden trabajar de forma equitativa y con calidad asistencial quirúrgica. Las variaciones por sexo en las EM post-quirúrgicas, una vez descartada la edad como indicador de gravedad, ponen de manifiesto la oportunidad de que los equipos quirúrgicos, mediante los diferentes estilos de práctica, de la evidencia científica y superación del desconocimiento, en sus sesiones y protocolos de actuación valoren las razones prequirúrgicas (sesgos de género en la derivación de pacientes), postquirúrgicas (sesgos de género en la valoración de la gravedad) y las consecuencias de los sesgos de género, como las reintervenciones, complicaciones tempranas y tardías, readmisiones y mortalidad.

\section{Agradecimientos}

Al Dr. Félix Lluís i Casajuana, Jefe del $S^{o}$ de Cirugía General y Aparato Digestivo HGUA. Al Dr. José Antonio Mayor, médico de la Unidad de Admisión y Documentación Clínica, HGUA. Al Dr. Carlos Álvarez-Dardet, Catedrático de Salud Pública de la Universidad de Alicante. 


\section{BIBLIOGRAFÍA}

- Aguilar, M. D.; Lázaro, P.; Fitch, K. y Luengo, S. (2002): “Gender differences in clinical status at time of coronary revascularisation in Spain”. En Journal Epidemiology Community Health, jul, vol. 56, nº, 7, pp. 555-559.

- Aguiló, L. J. (2005): “La complejidad de la interpretación de la variabilidad en cirugía general y digestiva”. En Atlas Var pract Med Sist Nac Salud, n. 2, pp. 79-80.

- Antevil, J.; Rivera, L.; Langenberg, B. y Brown, C. V. (2004): “The influence of age and gender on the utility of computed tomography to diagnose acute appendicitis". En The American Surgeon, vol. 70, nº 10, pp. 850-853.

- Attili, A. F. et al. (1995): "Epidemiology of Gallstone Disease in Italy: Prevalence Data of the Multicenter Italian Study on Cholelithiasis (M.I.COL.)". En American Journal of Epidemiology, vol. 141, $\mathrm{n}^{\circ}$. 2, pp. 158-165.

- Austin, P. C.; Rothwell, D. M. y Tu J. V. (2002): "Comparison of Statistical Modelling Strategies for Analyzing Length of Stay after CABG Surgery". En Health Services and Outcomes Research Methodology, vol. 3, n. 2, pp. 107-133.

- Badia, J. M. y Williamson, R. (1997): “Antibióticos e infección biliar”. En Cirugía Española, vol. 76, nº. 4, pp. 203-206.

- Baibergenova, A., et al. (2006): "Sex differences in hospital admissions from emergency departments in asthmatic adults: a population-based study". En Annals of Allergy, Asthma \& Immunology, may, vol. 96, n. 5, pp. 666-672.

- Barker, D. J. P.; Eriksson, J. G.; Forsen, T. y Osmond, C. (2005): "Infant growth and income 50 years later". Archives of Disease in Childhood, vol. 90, n. 3, pp. 272-273.

- Beresfold, A. A.; Weiss, N. S.; Voigt, L. F. y McKnight, B. (1997): "Risk of endometrial cancer in relation to use oestrogen combined with cyclic progestagen therapy in postmenopausal women”. En Lancet, vol. 349, pp. 458-461. 
- Bernard, A. M.; Hayward, R. A.; Rosevear, J. S. y McMahon, L. F. J. R. (1993): "Gender and hospital resource. Unexpected differences". En Evaluation \& The Health Professions, vol. 16, n. 2, pp. 177-189.

- Borkhoff, C. M. et al. (2008): “The effect of patients' sex on physicians' recommendations for total knee arthroplasty". En Canadian Medical Association Journal - CMAJ, vol. 178, nº. 6, pp. 681-687.

- Bucknall, T. E.; Cox, P. J. y Ellis, H. (1982): "Burst abdomen and incisional hernia: a prospective study of 1129 major laparotomies". En Britsh Medical Journal, vol. 284, nº 6320, pp. 931-933.

- Carbonell, A. M. et al. (2005): "Do patient or hospital demographics predict cholecystectomy outcomes?”. En Surgical Endoscopy, vol. 19, nº. 6, pp. 767-773.

- Elixhauser, A.; Steiner, C.; Harris, D. R. y Coffey, R. M. (1998): “Comorbidity measures for use with administrative data". En Medical Care, vol. 36, n. 1, pp. 8-27.

- Flum, D. R.; Morris, A.; Koepsell, T. y Dellinger, E. P. (2001): "Has misdiagnosis of appendicitis decreased over time? A population based analysis”. En The Journal of The American Medical Association - JAMA, vol. 286, nº. 13, pp. 1748-1753.

- Garavaglia, G. E. et al. (1989): "Sex differences in cardiac adaptation to essential hypertension”. En European Heart Journal, vol. 10, nº. 12, pp. 1110-1114.

- Gérvas, J. y Pérez Fernández, M. (1997): “Las hiperlipemias y la prevención primaria de la cardiopatía isquémica”. En Medicina Clínica (Barc), vol. 109, n. 14, pp. 549-552. - Gobierno Vasco (2000): Viceconsejeria de Planificación y Ordenación Sanitaria Departamento de Sanidad. Dirección de Planificación y Evaluación sanitaria. Informe del Registro de Altas Hospitalarias, [en línea] Disponible en:

http://www.osanet.euskadi.net/.../informacion/estado_salud/eu_5463/adjuntos/Informe_ hospitales_2000_3.pdf [Acceso 17/04/2007].

- González, J. R., et al. (2005): "Sex differences in hospital readmission among colorectal cancer patients". En Journal Epidemiology Community Health, vol. 59, nº 6, pp. 506-511. 
- Harding, K. G.; Mudge, M.; Leinster, S. J. y Hughes, L. E. (1983): “Late development of incisional hernia: an unrecognised problem”. En British Medical Journal, vo. 286, nº 6364, pp. 519-520.

- Hollenbeak, C. S.; Weisman, C. S.; Rossi, M. y Ettinger, S. M. (2006): “Gender Disparities in Percutaneous Coronary Interventions for Acute Myocardial Infarction in Pennsylvania”. En Medical Care, vol. 44, nº 1, pp. 24-30.

- Javitt, J. C., et al. (1995): "Geographic Variation in Utilization of Cataract Surgery". En Medical Care, vol. 33, nº. 1, pp. 90-105.

- Johnston, W. (1994): "Influence of sex on the results of abdominal aortic aneurysm repair”. En Journal of Vasculary Surgery, vol. 20, nº 6, pp. 914-926.

- Librero, J., et al. (2005): "Variaciones en intervenciones de Cirugía General en el Sistema Nacional de Salud”. En En Atlas Var pract Med Sist Nac Salud, no. 2, pp. 2-19. - López-Abente, O. G., et al. (2004): “Situación del cáncer en España: incidencia”. En Anales del Sistema Sanitario de Navarra, vol. 27, n. 2, pp.165-173.

- Malterud, K. (2000): "Symptoms as a source of medical knowledge: understanding medically unexplained disorders in women". En Family Medicine, vol. 32, no. 9, pp. 603-611.

- Marqués Espí, J. A.; Peiró, S. y Medrano Heredia, J. (2003): Variaciones en las tasa estandarizadas de intervenciones quirúrgicas en Alicante. Valencia: Escuela Valenciana de Estudios para la Salud.

- Martínez de Pancorbo, C., et al. (1997): "Prevalence and associated factors for gallstone disease: results of a population Survey in Spain". En Journal of Clinical Epidemiology, vol. 50, nº. 12, pp. 1347-1355.

- Ministerio de Ciencia y Innovación, España (2006): "Encuesta de Morbilidad Hospitalaria", [en línea] Disponible en: http://www.isciii.es/htdocs/centros/epidemiologia/morbilidad.jsp [Acceso 21/04/2010]. - Ministerio de Sanidad y Consumo, España (2008): “Clasificación Internacional de Enfermedades (9 $9^{a}$ Revisión- Modificación Clínica): eCIE-9-MC”, [en línea] Disponible en: http://www.msc.es/ecie9mc-2008/html/index.htm [Acceso 21/04/2010]. 
- ONU - Organización de Naciones Unidas (2001): “Informe de la Conferencia Mundial contra el Racismo, la Discriminación Racial, la Xenofobia y las Formas Conexas de Intolerancia", [en línea] Disponible en: http://www.unhchr.ch/huridocda/huridoca.nsf/(Symbol)/A.Conf.189.12.Sp?Opendocum ent [Acceso 21/04/2010].

- Ortún, V.; Puig-Junoy, J. y Callejón, M. (2005): “Innovación en medicamentos, precios y salud". En: González López-Valcárcel, B. (ed): Difusión en nuevas tecnologías sanitarias y políticas públicas. Barcelona: Masson, pp.173-193.

- Primatesta, P. y Goldacre, M. J. (1996): “Inguinal Hernia Repair: Incidence of Elective and Emergency Surgery, Readmission and Mortality". En International Journal of Epidemiology, vol. 25, nº. 4, pp. 835-839.

- Quan, H et al. (2005): "Coding algorithms for defining comorbidities in ICD-9-CM and ICD-10 administrative data". En Medicne Care, vol. 43, n. 11, pp. 1130-1139.

- Rajmil, L. et al. (1999): “Gender differences in children's hospitalization in Catalonia: another inequality?”. En Acta Paediatrica, vol. 88, n. 9, pp. 990-997.

- Ruiz, M. T. et al. (1995): “Atención según el género del paciente en los servicios de Urgencias: ¿diferente o desigual?”. En Gaceta Sanitaria, vol. 9, nº. 47, pp. 76-83.

- Ruiz-Cantero, M. T. y Verbrugge, L. (1997): “A two way view of gender bias in medicine”. En Journal Epidemiology Community Health, vol. 51, nº. 2, pp. 106-109.

- Ruiz-Cantero, M. T. y Verdú-Delgado, M. (2004): “Sesgo de género en el esfuerzo terapéutico". En Gaceta Sanitaria, vol. 18, sup. 1, 118-125.

- Ruiz-Cantero, M. T. et al. (2007): “A framework to analyse gender bias in epidemiological research". En Journal Epidemiology Community Health, vol. 61, n. 2, pp. 46-53.

- Ruiz López, P. et al. (2005): "Variabilidad de la práctica clínica en Cirugía General Estudios multicéntricos de la Asociación Española de Cirujanos: cirugía del cáncer colorrectal, colecistectomías y hernia inguinal”. En Atlas Var pract Med Sist Nac Salud, no. 2, pp. 55-59. 
- Sangha, O. et al. (2002): "Metric properties of the appropriateness evaluation protocol and predictors of inappropriate hospital use in Germany: an approach using longitudinal data". En International Journal for Quality in Health Care, vol. 14, n.6, pp. 483-492.

- Schwartzbauer, G. y Robbins, J. (2001): "Matters of Sex: Sex Matters". En Circulation, vol. 104, n. 12, pp. 1333-35.

- Schirmer, B. D.; Winters, K. L. y Edlich, R. F. (2005): "Cholelithiasis and cholecystitis". En Journal of Long-Term Effects of Medical Implants, vol. 15, n. 3, pp. 329-338.

- Tellado, J. M. et al. (2005): "Pautas de tratamiento antibiótico empírico de las infecciones intraabdominales". En Revista Española de Quimioterapia, vol. 18, nº 2, pp.179-186.

- Viñes, J. J.; Ardanaz, E.; Aráosla, A. y Gaminde, I. (2003): "Epidemiología clínica del cáncer colorrectal: revisión de la causalidad". En Anales del Sistema Sanitario de Navarra, vol. 26, no. 1, pp. 79-97.

- Zizza, C.; Herring, A.; Stevens, J. y Carey, T. (2003): "Bariatric Surgeries in North Carolina, 1990 to 2001: A Gender Comparison”. En Obesity Research, vol. 11, nº 12, pp. 1519-25. 
240 Arnold Bichler, C. B.; Ruiz Cantero, Ma. T.; Domínguez, J. T.; Gómez, V. C. y Segura, T. B.

\section{Tabla 1}

Frecuencias, Razón entre sexos y Tasas estandarizadas por sexo y edad de apendicectomías, intervenciones de hernias, colecistectomías y resecciones intestinales. 2000-2004. Servicio de Cirugía General y Digestiva. HGUA.

\begin{tabular}{|c|c|c|c|c|c|c|c|c|c|}
\hline & \multicolumn{2}{|c|}{ Sexo } & \multirow[t]{2}{*}{ Ratio H/M } & \multicolumn{4}{|c|}{ Tasa por sexo y edad $x 1^{-4}$ Habitantes } & \multirow[t]{2}{*}{$\mathbf{p}$} \\
\hline & & Hombre & Mujer & & Hombre & IC $95 \%$ & Mujer & IC $95 \%$ & \\
\hline \multicolumn{2}{|c|}{ Apendicectomías } & 576 & 397 & 1,45 & 12,57 & {$[11,54-13,6]$} & 8,15 & {$[7.34-8,95]$} & $<0.001$ \\
\hline \multicolumn{2}{|c|}{ Apendicitis Aguda } & 429 & 315 & 1,36 & 9,36 & {$[8,48-10,25]$} & 6,46 & {$[5,75-7,17]$} & $<0.001$ \\
\hline \multirow[t]{2}{*}{ Edad } & $15-34$ & 298 & 208 & 1,43 & 16,57 & {$[14,7-18,44]$} & 11,84 & {$[10,24-13,44]$} & $<0.001$ \\
\hline & $>35$ & 131 & 107 & 1,22 & 4,71 & {$[3,91-5,51]$} & 3,43 & {$[2,78-4,07]$} & 0.01 \\
\hline \multicolumn{2}{|c|}{$\begin{array}{c}\text { Apendicitis con } \\
\text { absceso intrabdominal } \\
\text { y/o Peritonitis }\end{array}$} & 147 & 82 & 1,79 & 3,21 & {$[2,69-3,73]$} & 1,68 & {$[1,32-2,05]$} & $<0.001$ \\
\hline \multirow[t]{2}{*}{ Edad } & $15-34$ & 67 & 44 & 1,52 & 3,73 & {$[2,84-4,62]$} & 2,51 & {$[1,77-3,25]$} & 0.04 \\
\hline & $>35$ & 80 & 38 & 2,1 & 2,87 & {$[2,24-3,49]$} & 1,22 & {$[0,84-1,6]$} & $<0.001$ \\
\hline \multicolumn{2}{|c|}{$\begin{array}{c}\text { Intervenciones de } \\
\text { Hernias }\end{array}$} & 1038 & 385 & 2,7 & 22,66 & {$[21,28-24,03]$} & 7,9 & {$[7,11-8,69]$} & $<0.001$ \\
\hline \multicolumn{2}{|c|}{$\begin{array}{c}\text { Hernias } \\
\text { Inguinocrurales }\end{array}$} & 896 & 170 & 5,27 & 19,56 & {$[18,28-20,84]$} & 3,49 & {$[2,96-4,01]$} & $<0.001$ \\
\hline \multirow[t]{2}{*}{ Edad } & $15-54$ & 344 & 67 & 5,13 & 10,3 & {$[9,22-11,38]$} & 2,01 & {$[1,52-2,49]$} & $<0.001$ \\
\hline & $>55$ & 552 & 103 & 5,35 & 44,5 & {$[40,8-48,2]$} & 6,67 & {$[5,24-8,1]$} & $<0.001$ \\
\hline \multicolumn{2}{|c|}{$\begin{array}{l}\text { Hernias Ventrales, } \\
\text { Incisionales y de } \\
\text { Pared }\end{array}$} & 142 & 215 & 0,66 & 3,1 & {$[2,59-3,61]$} & 4,41 & {$[3,82-5,00]$} & 0.001 \\
\hline \multirow[t]{2}{*}{ Edad } & $15-54$ & 58 & 60 & 0,96 & 1,74 & {$[1,29-2,18]$} & 1,8 & {$[1,34-2,25]$} & 0.9 \\
\hline & $>55$ & 84 & 155 & 0,54 & 6,77 & {$[5,33-8,21]$} & 10,04 & {$[8,47-11,61]$} & 0.004 \\
\hline \multicolumn{2}{|c|}{ Colecistectomías } & 458 & 722 & 0,63 & 10 & {$[9,08-10,91]$} & 14,81 & {$[13,73-15,89]$} & $<0.001$ \\
\hline \multicolumn{2}{|c|}{$\begin{array}{l}\text { Litiasis de vesícula y } \\
\text { vías biliares sin } \\
\text { colecistitis }\end{array}$} & 184 & 405 & 0,45 & 4,02 & {$[3,44-4,60]$} & 8,31 & {$[7,50-9,12]$} & $<0.001$ \\
\hline \multirow[t]{2}{*}{ Edad } & $15-54$ & 78 & 179 & 0,43 & 2,33 & {$[1,82-2,84]$} & 5,38 & {$[4,6-6,16]$} & $<0.001$ \\
\hline & $>55$ & 106 & 226 & 0,46 & 8,54 & {$[6,92-10,16]$} & 14,64 & {$[12,74-16,54]$} & $<0.001$ \\
\hline \multicolumn{2}{|c|}{$\begin{array}{l}\text { Litiasis de vesícula y } \\
\text { vías biliares con } \\
\text { colecistitis }\end{array}$} & 274 & 317 & 0,86 & 5,98 & {$[5,27-6,69]$} & 6,5 & {$[5,79-7,22]$} & 0.329 \\
\hline \multirow[t]{2}{*}{ Edad } & $15-54$ & 78 & 95 & 0,82 & 2,33 & {$[1,82-2,84]$} & 2,85 & {$[2,22-3,42]$} & 0.2 \\
\hline & $>55$ & 196 & 222 & 0,88 & 15,8 & {$[13,59-18,01]$} & 14,38 & {$[12,49-16,27]$} & 0.3 \\
\hline \multicolumn{2}{|c|}{$\begin{array}{c}\text { Resecciones por } \\
\text { Neoplasias } \\
\text { benignas y } \\
\text { malignas de } \\
\text { Intestino y Recto: }\end{array}$} & 212 & 186 & 1,14 & 4,63 & {$[4,01-5,25]$} & 3,82 & {$[3,27-4,36]$} & 0.06 \\
\hline \multirow[t]{3}{*}{ Edad } & $15-64$ & 67 & 59 & 1,13 & 1,73 & {$[1,32-2,14]$} & 1,52 & {$[1,14-1,9]$} & 0.5 \\
\hline & $65-75$ & 69 & 60 & 1,15 & 16,45 & {$[12,58-20,32]$} & 11,9 & {$[8,9-14,9]$} & 0.07 \\
\hline & $>75$ & 76 & 67 & 1,13 & 25,81 & {$[20,02-31,6]$} & 13,75 & {$[10,46-17,04]$} & $<0.001$ \\
\hline
\end{tabular}

Población tiempo quinquenio 2000-2004: 945.524 habitantes (458.118 H y 487.406 M). 
Tabla 2

Odds ratio crudo y ajustado por sexo y edad de apendicectomías, intervenciones de hernias, colecistectomías y resecciones intestinales. 2000-2004. Servicio de Cirugía General y Digestiva. HGUA.

\begin{tabular}{|c|c|c|c|c|c|c|c|c|c|c|c|c|}
\hline \multirow[b]{3}{*}{$\begin{array}{l}\text { Apendicec- } \\
\text { tomías }\end{array}$} & \multirow{2}{*}{\multicolumn{2}{|c|}{$\begin{array}{c}\text { Hombres } \\
\text { No Intervenidos }\end{array}$}} & \multirow{2}{*}{\multicolumn{2}{|c|}{$\begin{array}{c}\text { Mujeres } \\
\text { No } \\
\text { Intervenidas }\end{array}$}} & \multicolumn{4}{|c|}{ OR crudo (hombre-mujer) } & \multicolumn{4}{|c|}{$\begin{array}{c}\text { OR ajustado por edad } \\
\text { (basal hombres) }\end{array}$} \\
\hline & & & & & OR & IC & $\%)$ & $\mathbf{p}$ & OR & & $(95 \%)$ & $\mathbf{p}$ \\
\hline & 457.542 & 576 & 487.009 & 397 & 0,64 & 0,56 & 0,73 & $<0,001$ & 0,67 & 0,59 & 0,76 & $<0,001$ \\
\hline $\begin{array}{c}\text { Apendicitis } \\
\text { Aguda }\end{array}$ & 457.689 & 429 & 487.091 & 315 & 0,68 & 0,59 & 0,79 & $<0,001$ & 0,71 & 0,62 & 0,83 & $<0,001$ \\
\hline $\begin{array}{c}\text { Apendicitis } \\
\text { con } \\
\text { Absceso } \\
\text { intraabdom } \\
\text { inal y/o } \\
\text { Peritonitis }\end{array}$ & 457.971 & 147 & 487.324 & 82 & 0,52 & 0,40 & 0,68 & $<0,001$ & 0,53 & 0,40 & 0,69 & $<0,001$ \\
\hline $\begin{array}{c}\text { Intervencio } \\
\text { nes de } \\
\text { Hernias }\end{array}$ & 457.080 & 1.038 & 487.021 & 385 & 0,34 & 0,30 & 0,39 & $<0,001$ & 0,32 & 0,29 & 0,36 & $<0,001$ \\
\hline $\begin{array}{c}\text { Hernias } \\
\text { Inguino- } \\
\text { crurales }\end{array}$ & 457.222 & 896 & 487.236 & 170 & 0,17 & 0,15 & 0,20 & $<0,001$ & 0,16 & 0,14 & 0,19 & $<0,001$ \\
\hline $\begin{array}{c}\text { Hernia } \\
\text { Ventral, } \\
\text { Incisional } \\
\text { y de Pared }\end{array}$ & 457.976 & 142 & 487.191 & 215 & 1,42 & 1,15 & 1,75 & 0,001 & 1,31 & 1,06 & 1,62 & 0,01 \\
\hline $\begin{array}{l}\text { Colecistec- } \\
\text { tomías }\end{array}$ & 457.660 & 458 & 486.684 & 722 & 1,48 & 1,31 & 1,66 & $<0,001$ & 1.37 & 1.22 & $1.54 * * *$ & $<0,001$ \\
\hline $\begin{array}{l}\text { Litiasis de } \\
\text { vesícula y } \\
\quad \text { vías } \\
\text { biliares sin } \\
\text { colecistitis }\end{array}$ & 457.934 & 184 & 487.001 & 405 & 2,06 & 1,73 & 2,46 & $<0,001$ & 1,94 & 1,63 & 2,31 & $<0,001$ \\
\hline $\begin{array}{l}\text { Litiasis de } \\
\text { vesícula y } \\
\text { vías } \\
\text { biliares con } \\
\text { colecistitis }\end{array}$ & 457.844 & 274 & 487.089 & 317 & 1,08 & 0,92 & 1,27 & 0,3 & 0,99 & 0,84 & 1,16 & 0,9 \\
\hline $\begin{array}{c}\text { Resección } \\
\text { por } \\
\text { Neoplasias } \\
\text { benignas y } \\
\text { malignas } \\
\text { de } \\
\text { Intestino y } \\
\text { Recto }\end{array}$ & 457.906 & 212 & 487.220 & 186 & 0,82 & 0,67 & 1,00 & 0,06 & 0,69 & 0,56 & $0,84 * * *$ & 0,0002 \\
\hline
\end{tabular}

Fuente: CMBD 2000-2004. Unidad de Documentación Clínica y Admisión del HGUA y Tarjetas SIP Agencia Valenciana de Salut Departamento 19 Dirección Médica At Primaria. 


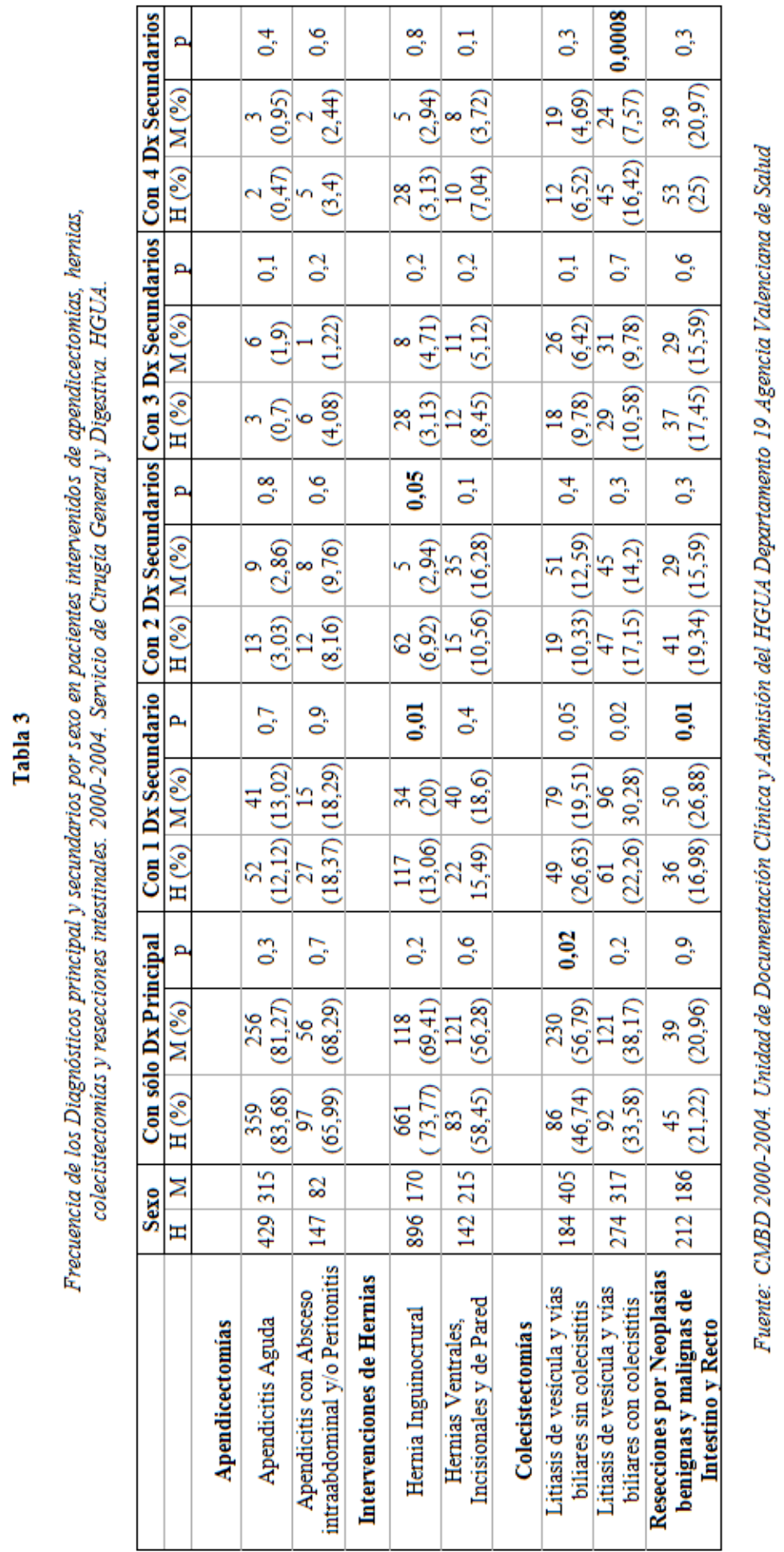


Tabla 4

Tipo de comorbilidad por sexo en pacientes intervenidos de apendicectomías, hernias, colecistectomías y resecciones intestinales. 2000-2004. Servicio de Cirugía General y Digestiva. HGUA.

\begin{tabular}{|c|c|c|c|}
\hline 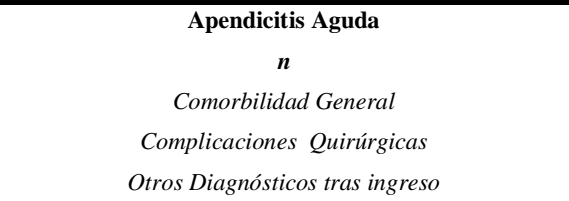 & $\begin{array}{l}\text { Hombres }(\%) \\
\begin{array}{cc} & 429 \\
26 & (6,1) \\
16 & (3,7) \\
6 & (1,4)\end{array}\end{array}$ & 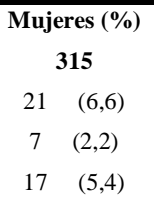 & $\begin{array}{c}0,7 \\
0,2 \\
\mathbf{0 , 0 0 1}\end{array}$ \\
\hline $\begin{array}{l}\text { Apendicitis con absceso Intrabdominal y/o Peritonitis } \\
\qquad \begin{array}{c}n \\
\text { Comorbilidad General } \\
\text { Complicaciones Quirúrgicas } \\
\text { Otros Diagnósticos tras ingreso }\end{array}\end{array}$ & \begin{tabular}{lc}
\multicolumn{2}{c}{147} \\
23 & $(15,6)$ \\
21 & $(14,3)$ \\
2 & $(1,4)$
\end{tabular} & $\begin{array}{cc} & \mathbf{8 2} \\
10 & (12,2) \\
5 & (6,1) \\
2 & (2,4)\end{array}$ & $\begin{array}{c}0,4 \\
0,06 \\
0,5\end{array}$ \\
\hline $\begin{array}{l}\text { Hernia Inguinocrural } \\
\qquad \\
\text { Comorbilidad General } \\
\text { Complicaciones Quirúrgicas } \\
\text { Otros Diagnósticos tras ingreso }\end{array}$ & $\begin{array}{cc}\mathbf{8 9 6} \\
225 & (25,1) \\
35 & (3,9) \\
5 & (0,5)\end{array}$ & \begin{tabular}{cc}
\multicolumn{2}{c}{$\mathbf{1 7 0}$} \\
45 & $(26,4)$ \\
7 & $(4,1)$ \\
-- & --
\end{tabular} & $\begin{array}{c}0,7 \\
0,8 \\
--\end{array}$ \\
\hline $\begin{array}{l}\text { Hernia Ventrales, Incisionales y Pared abdominal } \\
\qquad \boldsymbol{n} \\
\text { Comorbilidad General } \\
\text { Complicaciones Quirúrgicas } \\
\text { Otros Diagnósticos tras ingreso }\end{array}$ & $\begin{array}{cc} & 142 \\
58 & (40,8) \\
4 & (2,8) \\
4 & (2,8)\end{array}$ & $\begin{aligned} & 215 \\
85 & (39,5) \\
8 & (3,7) \\
6 & (2,8)\end{aligned}$ & $\begin{array}{l}0,8 \\
0,6 \\
0,9\end{array}$ \\
\hline 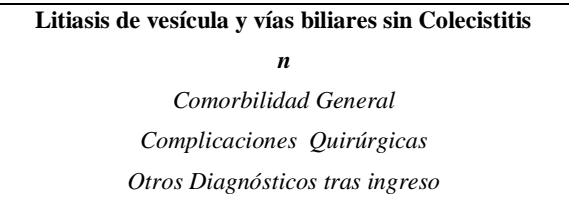 & $\begin{array}{cc} & 184 \\
105 & (57,06) \\
12 & (6,5) \\
30 & (16,3)\end{array}$ & \begin{tabular}{cc}
\multicolumn{2}{c}{405} \\
228 & $(56,3)$ \\
25 & $(6,2)$ \\
38 & $(9,4)$
\end{tabular} & $\begin{array}{c}0,9 \\
0,9 \\
\mathbf{0 , 0 1}\end{array}$ \\
\hline $\begin{array}{c}\text { Litiasis de vesícula y vías biliares con Colecistitis } \\
n \\
\text { Comorbilidad General } \\
\text { Complicaciones Quirúrgicas } \\
\text { Otros Diagnósticos tras ingreso } \\
\end{array}$ & \begin{tabular}{cc}
\multicolumn{2}{c}{$\mathbf{2 7 4}$} \\
230 & $(83,9)$ \\
43 & $(15,7)$ \\
33 & $(12,04)$ \\
\end{tabular} & \begin{tabular}{cc}
\multicolumn{3}{c}{$\mathbf{3 1 7}$} \\
222 & $(70,03)$ \\
26 & $(8,2)$ \\
60 & $(18,9)$ \\
\end{tabular} & $\begin{array}{c}<0,001 \\
\mathbf{0 , 0 0 4} \\
0,02\end{array}$ \\
\hline $\begin{array}{c}\text { Neoplasias Benignas y Malignas de Intestino y Recto } \\
\boldsymbol{n} \\
\text { Comorbilidad General } \\
\text { Complicaciones Quirúrgicas } \\
\text { Otros Diagnósticos tras ingreso } \\
\end{array}$ & \begin{tabular}{cc}
\multicolumn{2}{c}{$\mathbf{2 1 2}$} \\
164 & $(77,3)$ \\
54 & $(25,4)$ \\
90 & $(42,4)$
\end{tabular} & \begin{tabular}{cc}
\multicolumn{2}{c}{$\mathbf{1 8 6}$} \\
139 & $(74,7)$ \\
39 & $(20,9)$ \\
69 & $(37,09)$
\end{tabular} & $\begin{array}{l}0,2 \\
0,2 \\
0,5\end{array}$ \\
\hline
\end{tabular}

Fuente: CMBD 2000-2004. Unidad de Documentación Clínica y Admisión del HGUA Departamento 19 Agencia Valenciana de Salud 
244 Arnold Bichler, C. B.; Ruiz Cantero, Ma. T.; Domínguez, J. T.; Gómez, V. C. y Segura, T. B.

\section{Tabla 5}

Días de estancia media postquirúrgica, mediana y desviaciones, por apendicectomías, intervenciones de hernias, colecistectomías y resecciones intestinales. 2000-2004. Servicio de Cirugía General y Digestiva. HGUA.

\begin{tabular}{|c|c|c|c|c|c|c|c|c|}
\hline \multicolumn{4}{|c|}{ Frecuencia de Diagnósticos por edad y sexo } & \multicolumn{5}{|c|}{ EM postquirúrgica } \\
\hline & \multirow[b]{2}{*}{ Edad } & \multicolumn{2}{|c|}{$\mathbf{n}$} & \multicolumn{2}{|c|}{ Hombre } & \multicolumn{2}{|c|}{ Mujer } & p \\
\hline & & $\mathbf{H}$ & $\mathbf{M}$ & EM & M (DM) & EM & M (DM) & \\
\hline \multirow{2}{*}{ Apendicitis aguda } & $15-34$ & 298 & 208 & 3,76 & $3(1,39)$ & 3,5 & $3(1,3)$ & 0,1 \\
\hline & $>35$ & 131 & 107 & 5,61 & $5(2,44)$ & 4,77 & $4(1,9)$ & 0,1 \\
\hline $\begin{array}{l}\text { Apendicitis con } \\
\text { absceso }\end{array}$ & $15-34$ & 67 & 44 & 6,51 & $5(3,48)$ & 6,18 & $5(2,82)$ & 0,7 \\
\hline $\begin{array}{c}\text { intrabdominal } y / o \\
\text { peritonitis }\end{array}$ & $>35$ & 80 & 38 & 9,49 & $9(3,54)$ & 7,5 & $7(3,08)$ & 0,006 \\
\hline \multirow{2}{*}{$\begin{array}{c}\text { Hernias } \\
\text { inguinocrurales }\end{array}$} & $15-54$ & 344 & 67 & 1,47 & $1(1,3)$ & 1,22 & $1(1,12)$ & 0,7 \\
\hline & $>55$ & 552 & 103 & 3,36 & $2(2,42)$ & 3,88 & $3(2,44)$ & 0,007 \\
\hline \multirow{2}{*}{$\begin{array}{l}\text { Hernias ventrales, } \\
\text { incisionales y de } \\
\text { pared abdominal }\end{array}$} & $15-54$ & 58 & 60 & 4,57 & $2(3,74)$ & 5,93 & $5(3,4)$ & 0,001 \\
\hline & $>55$ & 84 & 155 & 7,78 & $6(4,71)$ & 7,06 & $6(3,66)$ & 0,6 \\
\hline \multirow{2}{*}{$\begin{array}{l}\text { Litiasis de vesícula } \\
\text { y vías biliares sin } \\
\text { colecistitis }\end{array}$} & $15-54$ & 78 & 179 & 4,32 & $3(2,4)$ & 3,78 & $3(1,79)$ & 0,4 \\
\hline & $>55$ & 106 & 226 & 6,19 & $5(3,36)$ & 6 & $4(3,51)$ & 0,2 \\
\hline \multirow{2}{*}{$\begin{array}{l}\text { Litiasis de vesícula } \\
\text { y vías biliares con } \\
\text { colecistitis }\end{array}$} & $15-54$ & 78 & 95 & 5,77 & $5(2,13)$ & 4,89 & $4(2,12)$ & $\mathbf{0 , 0 2}$ \\
\hline & $>55$ & 196 & 222 & 11,68 & $8(6,18)$ & 9,35 & $7(4,58)$ & 0,01 \\
\hline \multirow{3}{*}{$\begin{array}{c}\text { Resecciones por } \\
\text { Neoplasias } \\
\text { benignas y } \\
\text { malignas de } \\
\text { intestino y recto }\end{array}$} & $15-64$ & 67 & 59 & 15,57 & $10(7,9)$ & 13,46 & $10(6,3)$ & 0,4 \\
\hline & $65-74$ & 69 & 60 & 14,11 & $10(5,97)$ & 14,83 & $11(6,5)$ & 0,7 \\
\hline & $>75$ & 76 & 67 & 13,63 & $11(4,76)$ & 11,57 & $10(4,4)$ & 0,09 \\
\hline
\end{tabular}

Fuente: CMBD 2000-2004. Unidad de Documentación Clínica y Admisión del HGUA Departamento 19 Agencia Valenciana de Salud 


\section{ANEXO I}

Correspondencia entre Procedimientos quirúrgicos y Diagnóstico principal según la Clasificación Internacional de Enfermedades $9^{a}$ revisión Modificación Clínica.

\begin{tabular}{|c|c|c|c|c|}
\hline & CIE9MC de procedimiento & Códigos & $\begin{array}{c}\text { CIE9MC como Diagnóstico } \\
\text { principal }\end{array}$ & Códigos \\
\hline \multirow{3}{*}{$\begin{array}{c}\text { Cirugía } \\
\text { Apendicular }\end{array}$} & Apendicectomia laparoscópica & 47.01 & $\begin{array}{l}\text { Apendicitis Aguda sin } \\
\text { mención de peritonitis }\end{array}$ & 540.9 \\
\hline & Otra Apendicectomía & 47.09 & Apendicitis sin calificar & 541 \\
\hline & Otras Apendicectomías incidentales & 47.19 & Apendicitis con Absceso & 540.x \\
\hline \multirow{2}{*}{$\begin{array}{l}\text { Cirugía de vesícula } \\
\text { y vías biliares }\end{array}$} & Colecistectomía & 51.22 & $\begin{array}{c}\text { Litiasis Biliar y/o Vías } \\
\text { Biliares Con y Sin Colecistitis }\end{array}$ & 574.x \\
\hline & Colecistectomía Laparoscópica & 51.23 & Colecistitis & 575.x \\
\hline \multirow{11}{*}{$\begin{array}{l}\text { Cirugía de Hernias } \\
\text { Inguinocrurales }\end{array}$} & Reparación Unilateral de Hernia Inguinal & 53.00 & $\begin{array}{l}\text { Hernia Inguinal con y sin } \\
\text { obstrucción sin mención de } \\
\text { gangrena unilateral }\end{array}$ & $550 . \mathrm{x}$ \\
\hline & Reparación de Hernia Inguinal Directa & 53.01 & $\begin{array}{l}\text { Hernia femoral obstructiva } \\
\text { unilateral }\end{array}$ & 552.00 \\
\hline & Reparación de Hernia Inguinal Indirecta & 53.02 & Hernia femoral unilateral & 553.00 \\
\hline & $\begin{array}{l}\text { Reparación de hernia inguinal directa } \\
\text { con injerto o prótesis }\end{array}$ & 53.03 & & \\
\hline & $\begin{array}{l}\text { Reparación de hernia inguinal indirecta } \\
\text { con injerto o prótesis }\end{array}$ & 53.04 & & \\
\hline & $\begin{array}{c}\text { Reparación de hernia inguinal con injerto } \\
\text { o prótesis }\end{array}$ & 53.05 & & \\
\hline & $\begin{array}{l}\text { Repar. bilat. de hernia inguinal directa } \\
\text { con injerto o prótesis }\end{array}$ & 53.14 & & \\
\hline & $\begin{array}{l}\text { Repar. Bilat. de hernia inguinal con } \\
\text { injerto o prótesis }\end{array}$ & 53.17 & & \\
\hline & $\begin{array}{l}\text { Reparación Unilateral de hernia crural } \\
\text { con injerto o prot. }\end{array}$ & 53.21 & & \\
\hline & Otra herniorrafia unilateral & 53.29 & & \\
\hline & $\begin{array}{l}\text { Reparación bilateral de hernia crural con } \\
\text { injerto o prot. }\end{array}$ & 53.31 & & \\
\hline \multirow{4}{*}{$\begin{array}{l}\text { Cirugía de hernias } \\
\text { ventrales, } \\
\text { incisionales y de } \\
\text { pared abdominal }\end{array}$} & $\begin{array}{l}\text { Reparación de hernia incisional } \\
\text { (eventración) }\end{array}$ & 53.51 & $\begin{array}{l}\text { Hernia Ventral y otra hernia } \\
\text { ventral obstructiva por } \\
\text { incisión }\end{array}$ & 552.x \\
\hline & $\begin{array}{l}\text { Reparación de otra hernia de la pared } \\
\text { abdominal anterior }\end{array}$ & 53.59 & $\begin{array}{c}\text { Hernia Ventral y otra hernia } \\
\text { ventral }\end{array}$ & 553.x \\
\hline & $\begin{array}{c}\text { Reparación de hernia incisional con } \\
\text { prótesis }\end{array}$ & 53.61 & & \\
\hline & $\begin{array}{l}\text { Repar. de otra hernia de pared abdominal } \\
\text { anterior con prot }\end{array}$ & 53.69 & & \\
\hline \multirow[t]{6}{*}{$\begin{array}{c}\text { Cirugía de Intestino } \\
\text { y Recto }\end{array}$} & Otra excisión local de lesión de duodeno & 45.31 & $\begin{array}{l}\text { Neoplasia }(\mathrm{N}) \text { maligna de } \\
\text { otros sitios especificados del } \\
\text { estómago }\end{array}$ & 151.8 \\
\hline & $\begin{array}{l}\text { Otra elimin. o exéresis de lesión de Int. } \\
\text { Delgado, salvo duod }\end{array}$ & 45.34 & $\begin{array}{l}\text { N. maligna de Intestino } \\
\text { Delgado }\end{array}$ & 152.x \\
\hline & $\begin{array}{c}\text { Excisión local de lesión o tejido de } \\
\text { Intestino Grueso }\end{array}$ & 45.41 & $\begin{array}{l}\text { N. maligna de Intestino } \\
\text { Grueso }\end{array}$ & 153.x \\
\hline & $\begin{array}{c}\text { Polipectomía endoscópica del Intestino } \\
\text { Grueso }\end{array}$ & 45.42 & $\begin{array}{l}\mathrm{N} \text { maligna unión } \\
\text { rectosigmoidal }\end{array}$ & 154.0 \\
\hline & $\begin{array}{c}\text { Otra resección parcial de Intestino } \\
\text { Delgado }\end{array}$ & 45.62 & $\begin{array}{l}\text { N. maligna de peritoneo partes } \\
\text { especificadas }\end{array}$ & 158.8 \\
\hline & Resección total de intestino delgado & 45.63 & $\begin{array}{c}\mathrm{N} \text { maligna Tracto Intestinal } \\
\text { parte sin especificar }\end{array}$ & 159.0 \\
\hline
\end{tabular}


246 Arnold Bichler, C. B.; Ruiz, Cantero, Ma. T.; Domínguez, J. T.; Gómez, V. C. y Segura, T. B.

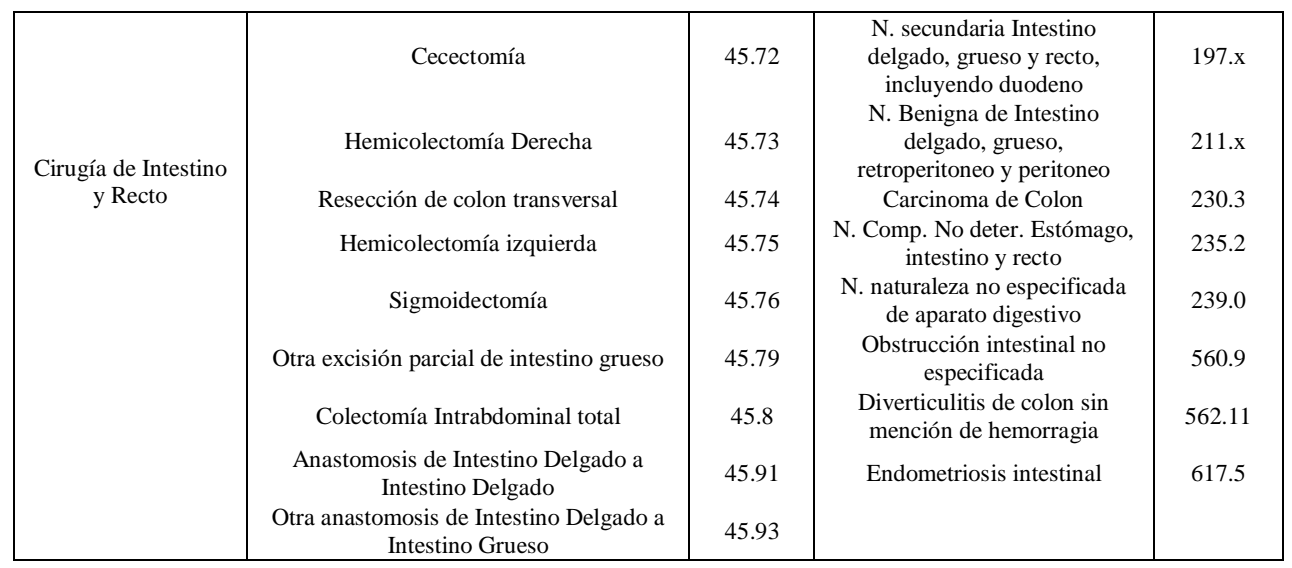

(x) Indica la selección de todos los posibles códigos con cualquier digito/s siguientes. 
ANEXO II
Descripción de los Diagnósticos Secundarios por Tipo de comorbilidad según sexo en pacientes intervenidos
de apendicectomías, hernias, y colecistectomías. 2000-2004. Servicio de Cirugía General y Digestiva. HGUA.

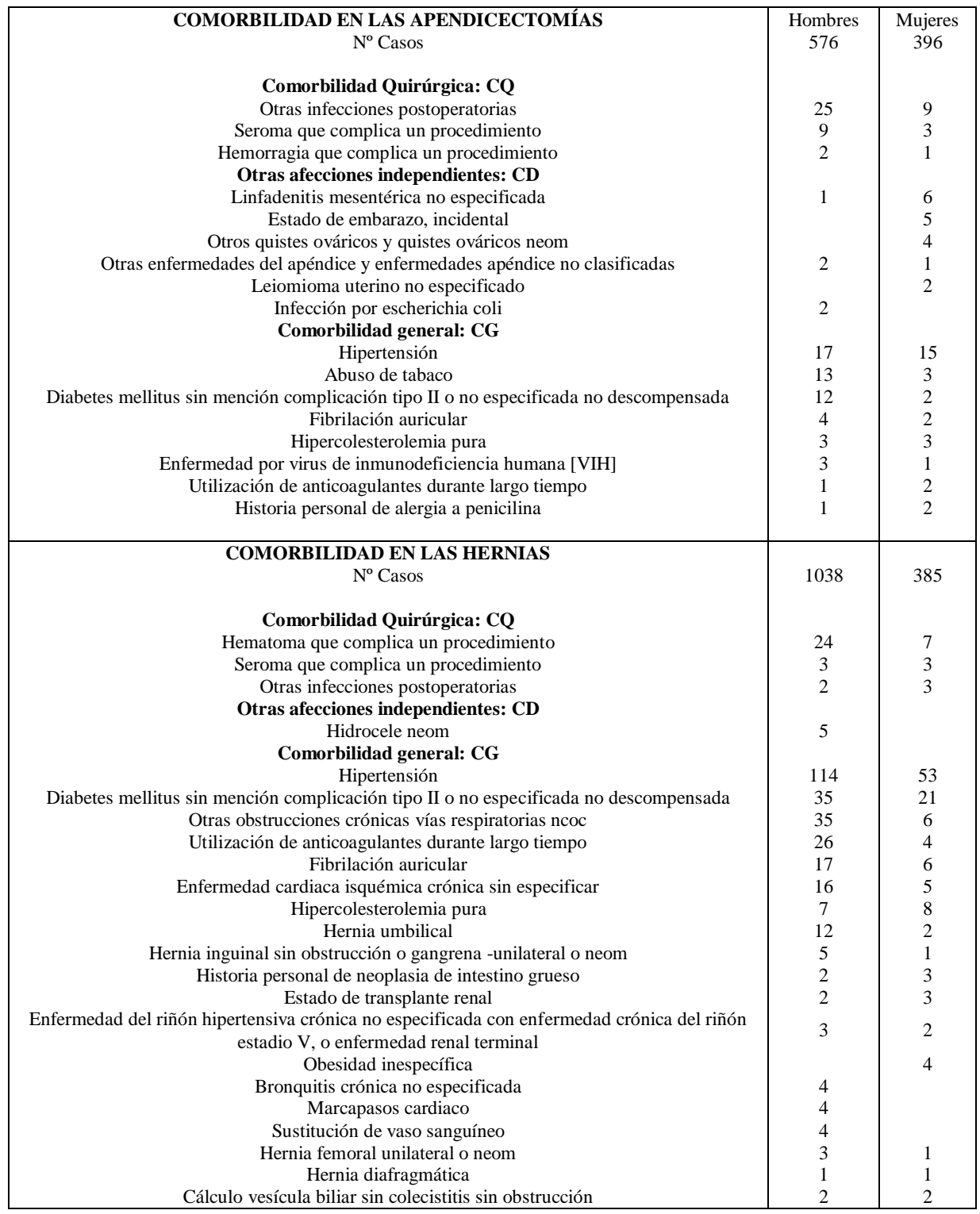


248 Arnold Bichler, C. B.; Ruiz Cantero, Ma. T.; Domínguez, J. T.; Gómez, V. C. y Segura, T. B.

\begin{tabular}{|c|c|c|}
\hline \multicolumn{3}{|l|}{ COMORBILIDAD EN LAS COLECISTECTOMÍAS } \\
\hline \multicolumn{3}{|l|}{ Comorbilidad Quirúrgica: CQ } \\
\hline Otras infecciones postoperatorias & 14 & 10 \\
\hline Procedimiento quirúrgico laparoscópico convertido en procedimiento abierto & 6 & 16 \\
\hline Hemorragia que complica un procedimiento & 6 & 3 \\
\hline Seroma que complica un procedimiento & 5 & 2 \\
\hline Fístula postoperatoria persistente & 3 & 4 \\
\hline Hematoma que complica un procedimiento & 3 & 1 \\
\hline Fístula de conducto biliar & 1 & 3 \\
\hline Otras afecciones independientes: CD & & \\
\hline Pancreatitis aguda & 33 & 55 \\
\hline Colesterolosis de vesícula biliar & 11 & 29 \\
\hline Pancreatitis crónica & 4 & 1 \\
\hline Calculo conducto biliar sin colecistitis sin obstrucción & 4 & 0 \\
\hline Colangitis & 4 & 4 \\
\hline \multicolumn{3}{|l|}{ Comorbilidad general: CG } \\
\hline Hipertensión & 116 & 156 \\
\hline Diabetes mellitus sin mención complicación tipo II o no especificada no descompensada & 50 & 66 \\
\hline Hipercolesterolemia pura & 13 & 22 \\
\hline Utilización de anticoagulantes durante largo tiempo & 14 & 21 \\
\hline Enfermedad cardiaca isquémica crónica sin especificar & 21 & 12 \\
\hline Fibrilación auricular & 13 & 19 \\
\hline Otras obstrucciones crónicas vías respiratorias ncoc & 12 & 5 \\
\hline Obesidad inespecífica & 1 & 14 \\
\hline Hernia diafragmática & 6 & 7 \\
\hline Hipotiroidismo no especificado & 1 & 9 \\
\hline Sustitución de válvula cardiaca & 0 & 6 \\
\hline Hernia inguinal sin obstrucción o gangrena -unilateral o neom & 4 & 2 \\
\hline $\begin{array}{c}\text { Enfermedad del riñón hipertensiva crónica no especificada con enfermedad crónica del riñón } \\
\text { estadio } \mathrm{V}, \mathrm{o} \text { enfermedad renal terminal }\end{array}$ & 2 & 3 \\
\hline Enfermedad cardiaca hipertensiva sin especificar sin fallo cardiaco congestivo & 1 & 4 \\
\hline Asma no especificada & 1 & 4 \\
\hline Estado de diálisis renal & 4 & 1 \\
\hline Hernia umbilical & 2 & 3 \\
\hline Estado de transplante renal & 1 & 4 \\
\hline Abuso de tabaco & 3 & 1 \\
\hline Hernia ventral por incisión & 3 & 1 \\
\hline Bronquitis crónica no especificada & 0 & 1 \\
\hline
\end{tabular}

Ncoc: no codificable bajo otro concepto - Neom: no especificado de otra manera 